\title{
BMJ Open Essential components of integrated care for youth with mental health and addiction needs: protocol for a scoping review
}

\author{
Cara A Settipani, ${ }^{1}$ Kristin Cleverley, ${ }^{1,2,3}$ Lisa D Hawke, ${ }^{1}$ Maureen Rice, ${ }^{4}$ \\ Joanna L Henderson ${ }^{1,3}$
}

To cite: Settipani CA, Cleverley K, Hawke LD, et al. Essential components of integrated care for youth with mental health and addiction needs: protocol for a scoping review. BMJ Open 2017;7:e015454. doi:10.1136/ bmjopen-2016-015454

- Prepublication history and additional material are available. To view, please visit the journal online (http://dx.doi.org/10. 1136/bmjopen-2016-015454).

Received 6 December 2016 Revised 8 February 2017 Accepted 31 March 2017

CrossMark

${ }^{1}$ Margaret and Wallace McCain Centre for Child, Youth and Family Mental Health, Centre for Addiction and Mental Health, Toronto, Ontario, Canada

${ }^{2}$ Lawrence S. Bloomberg Faculty of Nursing, University of Toronto, Toronto, Ontario, Canada

${ }^{3}$ Department of Psychiatry, University of Toronto, Toronto, Ontario, Canada

${ }^{4}$ Department of Clinical Epidemiology and Biostatistics, McMaster University, Hamilton, Ontario, Canada

Correspondence to

Dr Joanna L Henderson; joanna.henderson@camh.ca

\section{ABSTRACT}

Introduction Efforts to move towards integrated care have been met with increased interest and enthusiasm in recent years given the potential to improve care and population health while containing costs. However, there is a need to better understand community-based integrated care approaches for youth with mental health and/or addiction concerns to guide future implementation efforts and develop a set of standards for key components. The objectives of this scoping review are to: (1) identify the populations, settings, service providers, interventions, infrastructure and care coordination methods that have been included in integrated care for youth with mental health and/or addiction needs and (2) identify constructs that have been measured and evaluated (eg, outcomes, engagement) in the context of youth integrated care. Methods and analysis Seven electronic databases and several grey literature sources will be searched for material from 2001 to 2016 . Inclusion criteria will be broad with respect to type of work, as we will include all types of research studies as well as non-research studies that provide information relevant to characteristics and constructs measured in the context of integrated care for youth mental health. Titles and abstracts will be independently screened for eligibility by two raters using inclusion criteria. Full-text articles will then be accessed and independently screened for inclusion. A formal data extraction method will be employed, enabling synthesis of results in quantitative and qualitative formats.

Ethics and dissemination Results will be widely disseminated to various stakeholders to inform implementation and research efforts. Findings will also launch a Delphi method study leading to the development of an assessment tool for youth mental health services integration. This review does not require ethics approval.

\section{INTRODUCTION}

Mental health disorders are associated with substantial societal burden, ${ }^{1}$ yet issues with timely access to and availability of high-quality mental health services persist, particularly for youth. ${ }^{23}$ Integrated care has been identified as a way to improve health services with regard to access, quality, user satisfaction and

\section{Strengths and limitations of this study}

- This scoping review will provide a comprehensive review of integrated care addressing youth mental health and addiction in community settings, an unmet need in the literature.

- Information gleaned from this review will enable identification of key components of integrated care hubs and the development of a paradigm for integrated care for youth with mental health and addiction needs.

- The literature will be synthesised in quantitative and qualitative formats; however, scoping review methodology does not allow for assessing the quality of included work.

- Only English language publications will be included in this scoping review.

- The grey literature search for unpublished materials (eg, policy documents, agency reports) will be representative rather than exhaustive.

efficiency by linking together inputs, delivery, management and organisation of these services. ${ }^{4}$ Integrated care approaches have been recommended specifically for youth mental health given their potential to connect more youth with evidence-based interventions and improve outcomes. ${ }^{5}$ There is evidence supporting the benefits of team-based care in the context of collaborative care models for youth $^{6}$; however, more research specific to youth is needed. ${ }^{7}$ Importantly, little is known about what integrated care encompasses and how it has been implemented for youth with mental health and/or addiction needs. Integrated care delivery has been characterised as challenging due to the need to coordinate multiple systems and, frequently, restructure existing systems. ${ }^{8} 9$ Further complicating integration efforts, implementation of youth mental healthcare is associated with unique complexities. Youth are unlikely to effectively engage in and benefit from treatment 
without an informed, developmentally sensitive approach to the unique needs, relevant systems and barriers to care in this population. ${ }^{810-12}$ Improving access to and engagement with effective intervention is crucial given evidence that strategies to promote access and enhance treatment engagement are associated with better outcomes for youth $^{13}$ and research suggesting that successful treatment response in youth is associated with reduced risk for mental health and substance use problems in the long term. ${ }^{1415}$

Although there have been reviews of integrated care for adult populations ${ }^{16} 17$ as well as reviews and a meta-analysis focused on integration of behavioural health services in primary care settings, ${ }^{6}{ }^{18} 19$ there has not been a comprehensive review of integrated care addressing youth mental health and addiction in community settings. Recent efforts to create youthfriendly, integrated care hubs or one-stop shops for youth with mental health needs in community settings to reduce gaps in the health system and improve timely access to evidence-informed services ${ }^{20-22}$ suggest that a full picture of integrated care has not been captured. Despite the rapid adoption of this model worldwide, notably in Canada, the UK, Australia and Ireland, ${ }^{20-22}$ a clear understanding of the essential components of these integrated care efforts is lacking.

A comprehensive review of extant integrated care models and implementation efforts is needed to identify key components of integrated care hubs and create a paradigm for integrated care for youth with mental health and/or addiction needs. To this end, we will perform a scoping review to summarise the published and grey literature in this area. A scoping review was selected as appropriate because the methodology allows for comprehensively mapping and identifying the important concepts and literature available in a given research area in a structured manner. ${ }^{23}$ Given that we anticipate much of the work in this area has occurred in recent years, scoping review methodology will allow for an understanding of what evidence is available, including published and unpublished work. Other notable strengths of scoping reviews that led to the selection of this method include emphasis on the state of research activity and enabling policy makers to make evidence-informed decisions in a rapidly growing area. ${ }^{24}$

Objectives of the review are as follows: (1) identify the populations, settings, service providers, interventions, infrastructure and care coordination methods included in integrated care for youth with mental health and/ or addiction needs and (2) identify constructs, such as outcomes and treatment engagement, measured and evaluated in the context of youth integrated care. In addition to providing a necessary synthesis of current integrated care efforts for youth, these findings will inform the development of indicators for appraising integration of youth mental health services through a Delphi method study.

\section{METHODS AND ANALYSIS}

This scoping review will follow accepted methodology as detailed in the literature. ${ }^{24-27}$ The five-stage approach will be employed, namely (1) identifying the research questions; (2) identifying relevant studies; (3) selecting studies; (4) extracting data and (5) synthesising and reporting results.

\section{Stage 1: identifying the research questions}

Two broad research questions were developed that reflect the primary objectives of this exploratory scoping review:

1. What populations, settings, service providers, interventions, infrastructure and care coordination methods have been included in integrated care for youth with mental health and/or addiction needs?

2. What constructs have been specified, measured or evaluated in the context of youth integrated care?

\section{Stage 2: identifying relevant studies}

To capture the full breadth of work conducted in recent years across diverse contexts relevant to current integrated care efforts, all research studies and non-research literature, such as policy documents and agency reports, will be included in the review. Literature from 2001 to 2016 will be retained in order to capture the recent developments and current status of integrated care models. Eligible studies must address mental health, behavioural health or substance use concerns as a presenting problem in children, adolescents, youth, young adults or emerging adults. Work addressing concurrent disorders (cooccurring addiction and mental health problems) as a presenting problem will be included. A broad range of ages will be included, from 3 years of age through young adulthood (as defined in the identified literature), to ensure that work relevant to any developmental stage will be identified. Age will be examined in the data extraction phase given that models are likely to vary in meaningful ways as a function of ages served. To capture the integrated nature of service delivery, methods and models of care must bring together linked service components (eg, inputs, delivery, management, organisation of services) as a means of improving access, quality, user satisfaction, efficiency ${ }^{4}$ or outcomes ${ }^{5}$ and aim to make health systems or healthcare delivery more complete and comprehensive. ${ }^{28}$ These services must be integrated into the same setting and based in the community for inclusion in the present review.

\section{Search strategy}

A search, developed by an experienced medical research librarian, will be conducted in Medline, EMBASE, PsycINFO, CINAHL, Applied Social Sciences Index and Abstracts, Campbell Collaboration Library and Cochrane Library. It will combine subject heading and text word terms for integration AND mental/behavioural health AND children/adolescents. The search will be originally developed in Medline (see online supplementary appendix A) and then adapted with equivalent search terms appropriate for 
each database. Only English language publications will be included, from 2001 to 2016, and the search will exclude documents/notes, editorials and letters. In addition to the database search, the grey literature will be searched following a comprehensive methodological checklist. ${ }^{29}$ Essentially, a focused search will be performed on targeted child/adolescent mental health sites for unpublished materials that meet the inclusion criteria. Furthermore, Google Advanced Search will be used to undertake a broader search for grey literature. References of included studies will be checked for additional sources not found in the search and the International Journal of Integrated Care will be hand searched.

\section{Stage 3: study selection}

Inclusion criteria will be applied by two raters who will independently screen each title and abstract to determine eligibility (level 1). Reviewers will use the software program, Covidence, ${ }^{30}$ recommended by Cochrane for streamlining and improving the efficiency of systematic reviews. The project lead will regularly meet with reviewers throughout the title and abstract review process to discuss challenges that arise and refine the search strategy if necessary. After screening titles and abstracts, the fulltext articles deemed potentially eligible will be retrieved. Two raters will then complete level 2 review, examining full-text articles to determine whether they meet inclusion criteria. At both levels of review, the project lead will come to a decision on any disagreements that arise between the judgements of the two raters. The Preferred Reporting Items for Systematic Review and Meta-Analysis (see online supplementary PRISMA checklist) checklist will be followed and the four-phase PRISMA diagram will display search results and progression of studies screened, assessed for eligibility and ultimately included in synthesis. ${ }^{31}$

\section{Stage 4: data extraction}

The information to be obtained in the data extraction stage is shown in table 1.

1. Descriptive study information, including authors, title and citation, nature of work and whether it was retrieved from the published or grey literature, country and region of origin and scoping review research question addressed by the document.

2. Characteristics of integrated care models and implementation efforts, including a description of populations included, service settings, types of service providers, interventions employed or adapted, infrastructure and care coordination methods.

3. Constructs and outcomes; any constructs measured or described will be recorded as well as method of measurement, whether it was used as an outcome measure or to evaluate a component of integrated care, and how it indicates the goals of integrated care efforts.

In keeping with the methodology of scoping reviews, studies will not be evaluated on the basis of quality. ${ }^{25}$ Given that the process of selecting studies is intentionally iterative in nature, ${ }^{26}$ the data extraction tool will be amended based on knowledge attained during this process and modified as necessary. Similar to the method used in the study selection phase, the project lead will resolve discrepancies between the reviewers.

\section{Stage 5: synthesising and reporting results}

Information gleaned from the data extraction tool will be summarised quantitatively in table format. A map will be created based on number of articles to provide a visual

Table 1 Data extraction for the scoping review research questions

\section{Data to be extracted}

\begin{tabular}{|c|c|}
\hline Summary & $\begin{array}{l}\text { Author(s) } \\
\text { Title } \\
\text { Citation } \\
\text { Type of publication; published or grey } \\
\text { Location: country and region } \\
\text { Scoping review research question addressed }\end{array}$ \\
\hline $\begin{array}{l}\text { Research question 1: } \\
\text { populations, settings, } \\
\text { service providers, } \\
\text { interventions, infrastructure } \\
\text { and care coordination } \\
\text { methods }\end{array}$ & $\begin{array}{l}\text { Population characteristics (age, sex, other demographics, inclusion/exclusion criteria) } \\
\text { Type of service setting(s) } \\
\text { Titles of service providers and description of roles } \\
\text { Name and description of interventions and any adaptations } \\
\text { Infrastructure (eg, methods of sharing health records and care plans, health registries, } \\
\text { outcome tracking systems) } \\
\text { Care coordination methods }\end{array}$ \\
\hline
\end{tabular}


indicator of the current characteristics of integrated care for youth mental health and addiction and the constructs being examined and discussed in this area. Additionally, data will be analysed qualitatively to more thoroughly describe key concepts arising from the extant literature. As with other aspects of the scoping review methodology, synthesis procedures are also subject to refinement as the review progresses and additional knowledge is obtained.

\section{DISCUSSION AND DISSEMINATION}

The recent development of community-based integrated care hubs for youth with mental health and addiction concerns across several countries has the potential to meaningfully address longstanding problems with service delivery and access, yet a clearer understanding of key components of these models is needed. This scoping review will enable us to characterise the current focus of implementation efforts for youth integrated care in terms of the populations, settings, service providers, interventions, infrastructure and care coordination methods included. Identifying the constructs that have been discussed, measured and evaluated in the context of youth integrated care will inform evidence-based implementation of services and call attention to areas requiring further development. A better understanding of key components will also allow for formulating a clearer definition of the goals of integrated care for youth with mental health and/or addiction needs. Ultimately, results will guide the creation of indicators for a Delphi method study, which will produce a consensus statement and checklist that detail essential components of integrated care services addressing youth mental health and addiction.

Dissemination of findings will occur across various contexts to reach scientific, community, government and consumer groups. The project will be presented at the Canadian Psychological Association National Convention in 2017 and the 2017 Canadian Association for Health Services and Policy Research Conference, with the goals of disseminating findings to other professionals involved in advancing integrated care and soliciting feedback from those with expertise in this area. Findings will also be shared with our community partners who are actively involved in ongoing efforts to provide integrated care services to youth with mental health and addiction concerns. The Evidence Exchange Network for Mental Health and Addictions is an ideal format to disseminate research findings to a variety of stakeholders across Ontario and Canada including policy makers and system planners, and it is our hope that these findings will inform future efforts to create new integrated care hubs for youth in Canada. In addition, review results will be discussed with our youth engagement team, comprising youth with lived experience, to share knowledge with these important stakeholders and generate informed ideas for next needed steps. Finally, we will seek to publish results of this scoping review in an open access journal so that the information this review provides will be accessible to all those interested in improving integrated care for youth with mental health and addiction needs.

Acknowledgements We thank the YouthCan IMPACT Team for their support. KC held the CAMH Chair in Mental Health Nursing Research at the University of Toronto Lawrence S Bloomberg Faculty of Nursing and the Centre for Addiction and Mental Health during the preparation of this protocol.

Contributors CS, KC, LH, MR and JH contributed to the concept and design of the study. CS drafted the protocol and all authors (CS, KC, LH, MR and JH) contributed to the revision and finalisation of the manuscript. MR drafted the search strategy and conducted the literature search. All authors (CS, KC, LH, MR and JH) read and approved the final protocol. $\mathrm{JH}$ is the guarantor of this work.

Competing interests None declared.

Patient consent This study does not involve human subjects.

Provenance and peer review Not commissioned; externally peer reviewed.

Open Access This is an Open Access article distributed in accordance with the Creative Commons Attribution Non Commercial (CC BY-NC 4.0) license, which permits others to distribute, remix, adapt, build upon this work non-commercially, and license their derivative works on different terms, provided the original work is properly cited and the use is non-commercial. See: http://creativecommons.org/ licenses/by-nc/4.0/

(c) Article author(s) (or their employer(s) unless otherwise stated in the text of the article) 2017. All rights reserved. No commercial use is permitted unless otherwise expressly granted.

\section{REFERENCES}

1. Ratnasingham S, Cairney J, Manson $\mathrm{H}$, et al. The burden of mental illness and addiction in Ontario. Can J Psychiatry 2013;58:529-37.

2. Tylee A, Haller DM, Graham T, et al. Youth-friendly primary-care services: how are we doing and what more needs to be done? Lancet 2007;369:1565-73.

3. Wang PS, Lane M, Olfson M, et al. Twelve-month use of mental health services in the United States: results from the National Comorbidity Survey replication. Arch Gen Psychiatry 2005;62:629-40.

4. Gröne O, Garcia-Barbero M. Integrated care: a position paper of the WHO European office for integrated health care services. Int $J$ Integr Care 2001;1:e21.

5. de Voursney D, Huang LN. Meeting the mental health needs of children and youth through integrated care: a systems and policy perspective. Psychol Serv 2016;13:77-91.

6. Asarnow JR, Rozenman M, Wiblin J, et al. Integrated medicalbehavioral care compared with usual primary care for child and adolescent behavioral health: a meta-analysis. JAMA Pediatr 2015;169:929-37.

7. Asarnow JR, Hoagwood KE, Stancin T, et al. Psychological science and innovative strategies for Informing health care redesign: a policy brief. J Clin Child Adolesc Psychol 2015;44:923-32.

8. McGorry P, Bates T, Birchwood M. Designing youth mental health services for the 21st century: examples from Australia, Ireland and the UK. Br J Psychiatry 2013;54(s54):s30-s35.

9. Nicaise P, Tulloch S, Dubois V, et al. Using social network analysis for assessing mental health and social services inter-organisational collaboration: findings in deprived areas in Brussels and London. Adm Policy Ment Health 2013;40:331-9.

10. Kendall PC, Peterman JS. CBT for Adolescents with anxiety: mature yet still developing. Am J Psychiatry 2015;172:519-30.

11. Sylwestrzak A, Overholt CE, Ristau KI, et al. Self-reported barriers to treatment engagement: adolescent perspectives from the National Comorbidity Survey-Adolescent supplement (NCS-A). Community Ment Health J 2015;51:775-81.

12. Green CA, Wisdom JP, Wolfe L, et al. Engaging youths with serious mental illnesses in treatment: stars study consumer recommendations. Psychiatr Rehabil J 2012;35:360-8.

13. Lindsey MA, Brandt NE, Becker KD, et al. Identifying the common elements of treatment engagement interventions in children's mental health services. Clin Child Fam Psychol Rev 2014;17:283-98.

14. Benjamin CL, Harrison JP, Settipani CA, et al. Anxiety and related outcomes in young adults 7 to 19 years after receiving treatment for child anxiety. J Consult Clin Psychol 2013;81:865-76. 
15. Kendall PC, Safford S, Flannery-Schroeder E, et al. Child anxiety treatment: outcomes in adolescence and impact on substance use and depression at 7.4-year follow-up. J Consult Clin Psychol 2004;72:276-87.

16. Happell B, Galletly C, Castle D, et al. Scoping review of research in Australia on the co-occurrence of physical and serious mental illness and integrated care. Int J Ment Health Nurs 2015;24:421-38.

17. Chen S, Awan S, Rajji T, et al. Integrated care pathways for schizophrenia: a scoping review. Adm Policy Ment Health 2016;43:760-7.

18. Martin MP, White MB, Hodgson JL, et al. Integrated primary care: a systematic review of program characteristics. Fam Syst Health 2014;32:101-15.

19. Njoroge WF, Hostutler CA, Schwartz BS, et al. Integrated behavioral health in pediatric primary care. Curr Psychiatry Rep 2016;18:106.

20. McGorry PD, Tanti C, Stokes R, et al. Headspace: Australia's National Youth Mental Health Foundation--where young minds come first. Med J Aust 2007;187(7 Suppl):S68-70.

21. Bates T, Illback R, Scanlan F, et al. Somewhere to turn to, someone to talk to. Dublin 2009

22. Henderson JL, Cheung A, Cleverley K, et al. Integrated collaborative care teams to enhance service delivery to youth with mental health and substance use challenges: protocol for a pragmatic randomised controlled trial. BMJ Open 2017; 7: e014080.
23. Canadian Institutes of Health Research. A guide to knowledge synthesis, 2010.

24. O'Brien KK, Colquhoun $\mathrm{H}$, Levac D, et al. Advancing scoping study methodology: a web-based survey and consultation of perceptions on terminology, definition and methodological steps. BMC Health Serv Res 2016;16:305.

25. Arksey H, O'Malley L. Scoping studies: towards a methodological framework. Int J Soc Res Methodol 2005;8:19-32.

26. Levac D, Colquhoun H, O'Brien KK. Scoping studies: advancing the methodology. Implement Sci 2010;5:69.

27. Peters MD, Godfrey CM, Khalil H, et al. Guidance for conducting systematic scoping reviews. Int J Evid Based Healthc 2015;13:141-6.

28. Kodner DL, Spreeuwenberg C. Integrated care: meaning, logic, applications, and implications--a discussion paper. Int J Integr Care 2002;2:e12.

29. Canadian Agency for Drugs and Technology in Health. Grey matters: a practical search tool for evidence-based medicine, (accessed 1 Dec 2015).

30. Covidence systematic review software, Veritas Health Innovation, Melbourne, Australia.

31. Moher D, Liberati A, Tetzlaff J, et al. Preferred reporting items for systematic reviews and meta-analyses: the PRISMA statement. PLOS Med 2009;6:e1000097. 